\title{
Esophageal Anastomotic Leakage
}

National Cancer Institute

\section{Source}

National Cancer Institute. Esophageal Anastomotic Leakage. NCI Thesaurus. Code

C78270.

Leakage due to breakdown of an esophageal anastomosis. 